\title{
Efficacy and safety
}

of artemether-lumefantrine for the treatment of uncomplicated malaria and prevalence of Pfk 13 and Pfmdr 1 polymorphisms after a decade of using artemisinin-based combination therapy in mainland Tanzania

Deus S. Ishengoma ${ }^{1 *} \mathbb{0}$, Celine I. Mandara ${ }^{1,7}$, Filbert Francis ${ }^{1}$, Eldin Talundzic², Naomi W. Lucchi², Billy Ngasala ${ }^{3,4}$, Abdunoor M. Kabanywanyi ${ }^{5}$, Muhidin K. Mahende ${ }^{5}$, Erasmus Kamugisha ${ }^{6}$, Reginald A. Kavishe ${ }^{7}$, Florida Muro ${ }^{7}$, Ally Mohamed ${ }^{8}$, Renata Mandike ${ }^{8}$, Sigsbert Mkude ${ }^{8}$, Frank Chacky ${ }^{8}$, Lynn Paxton², George Greer ${ }^{9}$,

Chonge A. Kitojo ${ }^{9}$, Ritha Njau ${ }^{10}$, Troy Martin ${ }^{11}$, Meera Venkatesan ${ }^{12}$, Marian Warsame ${ }^{13,15}$, Eric S. Halsey 2,14 and Venkatachalam Udhayakumar ${ }^{2}$

\begin{abstract}
Background: The World Health Organization recommends regular therapeutic efficacy studies (TES) to monitor the performance of first and second-line anti-malarials. In 2016, efficacy and safety of artemether-lumefantrine (AL) for the treatment of uncomplicated falciparum malaria were assessed through a TES conducted between April and October 2016 at four sentinel sites of Kibaha, Mkuzi, Mlimba, and Ujiji in Tanzania. The study also assessed molecular markers of artemisinin and lumefantrine (partner drug) resistance.

Methods: Eligible patients were enrolled at the four sites, treated with standard doses of AL, and monitored for 28 days with clinical and laboratory assessments. The main outcomes were PCR corrected cure rates, day 3 positivity rates, safety of AL, and prevalence of single nucleotide polymorphisms in Plasmodium falciparum kelch 13 (Pfk13)

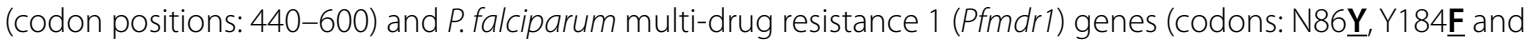
D1246Y), markers of artemisinin and lumefantrine resistance, respectively.

Results: Of 344 patients enrolled, three withdrew, six were lost to follow-up; and results were analysed for 335 (97.4\%) patients. Two patients had treatment failure (one early treatment failure and one recrudescent infection) after PCR correction, yielding an adequate clinical and parasitological response of $>98 \%$. Day 3 positivity rates ranged from 0 to 5.7\%. Common adverse events included cough, abdominal pain, vomiting, and diarrhoea. Two patients had serious adverse events; one died after the first dose of $\mathrm{AL}$ and another required hospitalization after the second dose of AL (on day 0) but recovered completely. Of 344 samples collected at enrolment (day 0), $92.7 \%$ and $100 \%$ were successfully sequenced for Pfk 13 and Pfmdr1 genes, respectively. Six (1.9\%) had non-synonymous mutations in Pfk13, none of which had been previously associated with artemisinin resistance. For Pfmdr1, the NED haplotype (codons
\end{abstract}

\footnotetext{
*Correspondence: deusishe@yahoo.com

${ }^{1}$ National Institute for Medical Research, Tanga Research Centre, Tanga,

Tanzania

Full list of author information is available at the end of the article
} 
N86, 184F and D1246) was detected in 134 (39.0\%) samples; ranging from 33.0\% in Mlimba to 45.5\% at Mkuzi. The difference among the four sites was not significant $(p=0.578)$. All samples had a single copy of the Pfmdr 1 gene.

Conclusion: The study indicated high efficacy of AL and the safety profile was consistent with previous reports. There were no known artemisinin-resistance Pfk 13 mutations, but there was a high prevalence of a Pfmdr 1 haplotype associated with reduced sensitivity to lumefantrine (but no reduced efficacy was observed in the subjects). Continued TES and monitoring of markers of resistance to artemisinin and partner drugs is critical for early detection of resistant parasites and to inform evidence-based malaria treatment policies.

Trial Registration ClinicalTrials.gov NCT03387631

Keywords: Efficacy, Safety, Artemether-lumefantrine, Falciparum malaria, Tanzania

\section{Background}

Despite a decline of malaria burden over the past decade, malaria remains a major public health threat $[1,2]$. An estimated 435,000 deaths and over 219 million cases were reported in 2017 (an increase of 5 and 3 million cases compared to 2015 and 2016, respectively) [1]. Approximately $93 \%$ of the deaths and $92 \%$ of the cases were from sub-Saharan Africa, with the majority occurring in children under 5 years of age or pregnant women [1]. Malaria control relies on a handful of interventions, including prompt and effective treatment with anti-malarials [3], a strategy threatened by parasite resistance to anti-malarial drugs in Southeast Asia [4].

Artemisinin-based combination therapy ACT is recommended by the World Health Organization (WHO) for the treatment of uncomplicated malaria caused by Plasmodium falciparum [5]. The currently recommended combinations include artemether-lumefantrine $(\mathrm{AL})$, artesunate-amodiaquine (ASAQ), artesunatemefloquine, dihydroartemisinin-piperaquine (DP) and artesunate-sulfadoxine/pyrimethamine (AS+SP) [6]. Tanzania introduced AL as its first line drug for the treatment of uncomplicated falciparum malaria in 2006 [7], and it remains the sole first-line treatment recommended in the country [8]. Studies conducted in the East African countries of Tanzania [9-12], Kenya [13], Uganda [14], Rwanda [15], Burundi [16], and in other African countries [17-19] have shown that AL, as well as other artemisinin-based combinations, such as ASAQ and DP (which are first or second-line therapies in other African countries) have high therapeutic efficacy and are well tolerated with minimal adverse effects. To date, there are no reports of clinically significant artemisinin resistance in Africa, and the laboratory correlates of resistance in Southeast Asia, delayed parasite clearance [20] or Plasmodium falciparum kelch 13 (Pfk13) gene mutations [21-23] are rare in Africa. Studies from Southeast Asia have demonstrated resistance to partner drugs, including piperaquine [22, 24], yielding parasites resistant to both components of ACT, a scenario fortunately not currently observed in Africa.
Due to the threat of artemisinin drug resistance, WHO recommends regular surveillance (biennial) to monitor the performance of anti-malarials in malaria endemic countries. Furthermore, the WHO recommends molecular surveillance based on known polymorphisms (including copy number variations) in the P. falciparum genome as markers of resistance to artemisinins and partner drugs of the currently used artemisinin-based combinations. A number of single nucleotide polymorphisms (SNPs) in the Pfk13 gene have been shown to confer resistance to artemisinins [25, 26]; and a number

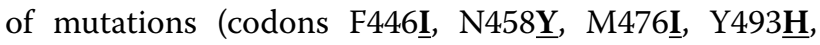
R539T, I543ㅈ, P553 $\underline{\mathbf{L}}, \mathrm{R} 561 \underline{\mathrm{H}}$ and C580Y) have been validated as markers of parasite resistance to artemisinins in Southeast Asia [27]. Polymorphisms in the P. falciparum multidrug resistance 1 ( $P f m d r 1$ ) gene appear to be associated with decreased susceptibility to lumefantrine [28, 29]. Gene duplication leading to increased Pfmdr1 copy numbers has been suggested to be associated with reduced susceptibility to lumefantrine [30].

In Tanzania, the National Malaria Control Programme (NMCP) and its partners have been implementing therapeutic efficacy studies (TESs) to monitor the efficacy and safety of different anti-malarials [31, 32], including currently used artemisinin-based combinations or of potential use in the country [9-12]. The studies have been conducted at eight sentinel sites located in regions with different transmission intensity, with some in border areas possessing potential for introduction of parasites from neighbouring countries [31]. Independent researchers have also conducted similar studies at the NMCP sentinel sites or other study areas [9]. These data supported changes in national anti-malarial treatment guidelines to replace chloroquine with SP in 2001 [33] and SP with AL in 2006 [7]. The study reported herein was carried out to assess the efficacy and safety of AL for the treatment of uncomplicated falciparum malaria, and the prevalence of molecular markers known to be associated with artemisinin resistance and reduced susceptibility to the partner drug (lumefantrine) after using AL in Tanzania for 10 years. 


\section{Methods}

\section{Study sites}

This study was carried out at four of the eight NMCP sentinel sites (Kibaha-Coast region, Mkuzi-Tanga, Mlimba-Morogoro, and Ujiji-Kigoma) between April and September 2016. The study sites (Fig. 1) have been NMCP sentinel sites for monitoring of anti-malarial efficacy since 1997 [31, 32].

In Kibaha, the study was conducted at Yombo Health Centre, which is located in Kibaha district of Coastal region (Pwani), about $100 \mathrm{~km}$ west of Dar es Salaam (the commercial capital of Tanzania). Kibaha has low malaria transmission $(<10 \%)$ as reported in previous population surveys [34-36]. Malaria transmission in Kibaha occurs throughout the year and peaks during or just after the rainy season.

Mkuzi health centre is located in Muheza district of Tanga region, in northeastern Tanzania. The malaria epidemiological profile of Muheza district has been well characterized and a detailed description given elsewhere [37, 38]. Mkuzi is also one of the sentinel sites with low malaria transmission in Tanzania with parasite

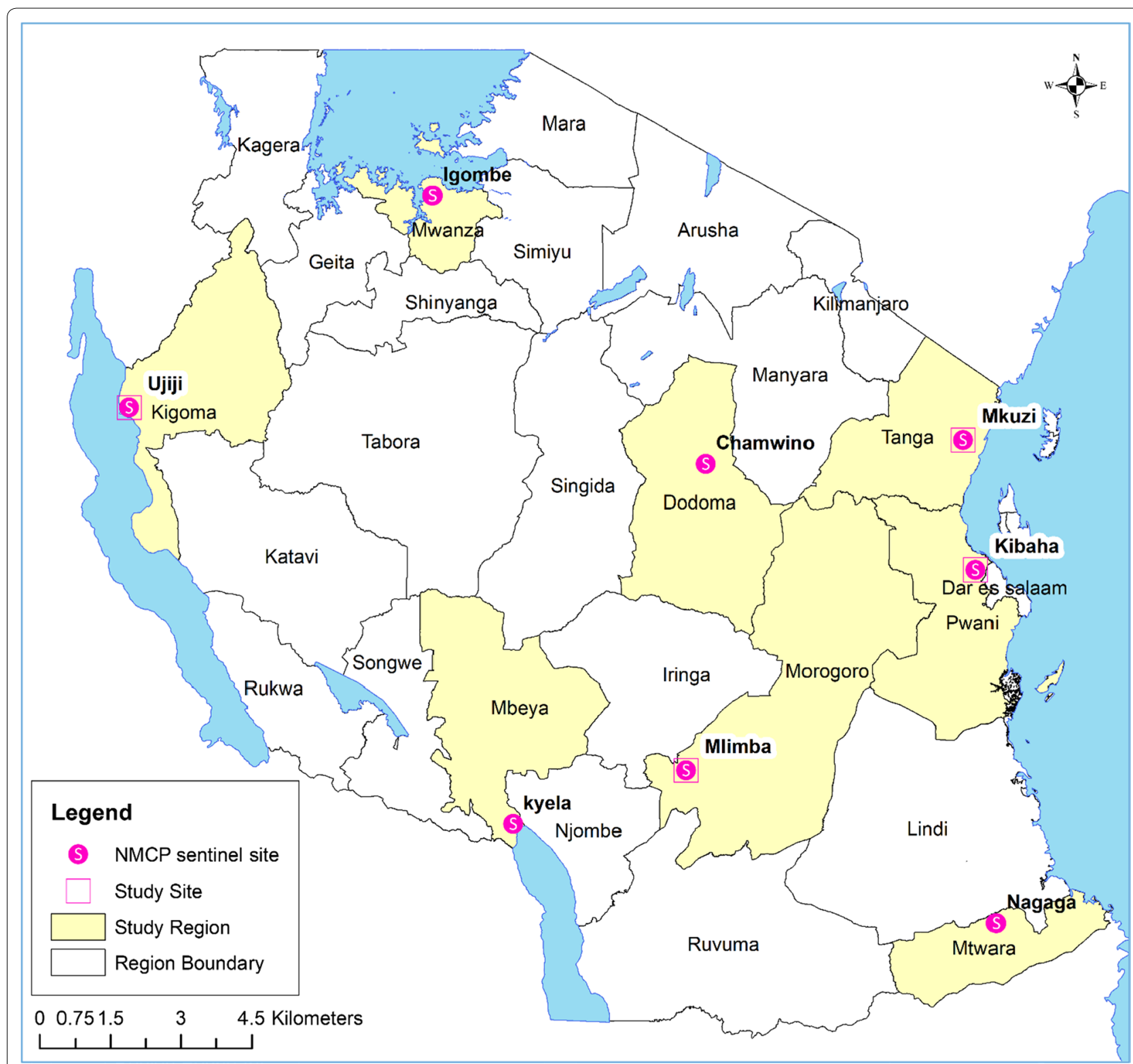

Fig. 1 Regional map of Tanzania with eight National Malaria Control Programme sentinel sites, with the four sites covered in the 2016 study marked with white squares 
prevalence of $<5 \%$, as reported in a 2017 national survey [39]. Details of the site as well as Muheza have been recently covered elsewhere [40].

Ujiji health centre is located in Kigoma urban district of Kigoma region, on the eastern shores of Lake Tanganyika in the northwestern part of Tanzania. Despite a decline in malaria transmission reported from 2007 onwards [34-36, 39], overall malaria transmission in Kigoma has increased in the past decade. According to recent national surveys, parasite prevalence increased from its 2007 level of $19.6 \%$ to $38.1 \%$ in 2016 , followed by a decline to $24.4 \%$ in 2017 , which was the highest prevalence in the country [34-36, 39]. Further details about Ujiji site have been given elsewhere [40].

Mlimba health centre is located in Kilombero district of Morogoro region and the areas around this site have been extensively studied under the Ifakara Health Institute demographic surveillance system over the past two decades [41-43]. Kilombero had high malaria transmission, with parasite prevalence of about $70.0 \%$ and entomological inoculation rates (EIR) of $>300$ infectious bites per person per year in the 1990s [44]. However, malaria transmission in Kilombero has recently declined due to different interventions, and it is now a low transmission area (prevalence $<10 \%$ in 2017) [39].

\section{Study design and study population}

This was a single-arm prospective in vivo study designed to assess the therapeutic efficacy and safety of AL for the treatment of uncomplicated falciparum malaria and the markers of artemisinin and lumefantrine resistance. Malaria transmission has been decreasing in some of the sites compared to the past and, therefore, thresholds of parasitaemia for low transmission areas recommended by WHO (between 250 and 200,000 asexual parasites/ $\mu \mathrm{l})$ were used. The age of study participants ( 6 months to 10 years) was also broadened $[45,46]$.

\section{Sample size estimation}

Per the WHO protocol, sample size estimates assumed $5 \%$ of the enrolled patients would have treatment failure after treatment with AL. At a confidence level of $95 \%$ and an estimate precision of $5 \%$, a minimum sample size of 73 patients was required to detect a failure rate $\leq 5 \%$ [46]. With a $20 \%$ increase to allow for loss to follow-up and withdrawals during the 28-days of follow-up, 88 patients were targeted per site, giving a total of 352 at the four sites.

\section{Screening and recruitment}

At each of the study sites, potential participants were screened at the outpatient departments using malaria rapid diagnostic tests and microscopy as previously described [40]. Patients were eligible for enrolment if they were aged 6 months to 10 years, had fever at presentation (axillary temperature $\geq 37.5^{\circ} \mathrm{C}$ ) and/or history of fever in the last $24 \mathrm{~h}$, a positive rapid diagnostic test, and parasitaemia of 250 to 200,000 asexual parasites/ $\mu$ l by microscopy. Other inclusion and exclusion criteria were assessed according to the WHO protocol [46]. Patients who could not be enrolled in the study received appropriate treatment according to national guidelines [8]. In addition to the malaria parasite identification, dried blood spots (DBS) on filter paper (Whatmann No. 3, GE Healthcare Life Sciences, PA, USA) were collected for parasite genotyping (to distinguish recrudescence from new infections) and for analysis of markers of artemisinin and lumefantrine resistance. Microscopy was performed during each subsequent visit to determine infection status, species, and parasite density.

\section{Examination of malaria parasites by microscopy}

Two blood slides were collected, and one of the slides was stained with $10 \%$ Giemsa for 10-15 min and examined by microscopy to detect presence of and an estimated density of malaria parasites. The second blood slide was stained with $3 \%$ Giemsa for $30-45 \mathrm{~min}$ and used to determine the actual parasite density, species, and presence of gametocytes. Parasitaemia was measured by counting the number of asexual parasites against 200 leucocytes in thick blood films and detection of the different parasite species was done on thin films. Parasite density per $\mu \mathrm{l}$ of blood was calculated by multiplying the total count by 40 , assuming that $1 \mu \mathrm{l}$ of blood had a mean count of 8000 leucocytes [26]. When more than 500 parasites were identified before counting 200 leucocytes, counting was stopped and parasitaemia was calculated using the actual number of leucocytes counted. A blood slide was declared negative when examination of 100 high power fields did not reveal the presence of malaria parasites. For quality control, each slide was re-examined by a second microscopist, and those with discrepant results were reexamined by a third microscopist. Any further disagreement was resolved by a team of three microscopists, who examined the same slide at the same time. Final parasitaemia was calculated as the average between the two closest readings.

\section{Treatment and clinical monitoring during follow-up} Enrolled patients were treated with $\mathrm{AL}\left(\mathrm{Coartem}^{\circledR}\right.$, Beijing Novartis Pharma Ltd, Beijing China; provided by WHO) for 3 days. Weight-based dosing based on one of three ranges $(5-14 \mathrm{~kg}, 15-24 \mathrm{~kg}$, or $25-35 \mathrm{~kg})$ was done using a fixed dose combination of $20 \mathrm{mg}$ of artemether and $120 \mathrm{mg}$ lumefantrine per tablet, thus patients were given one, two or three tablets from smallest to heaviest, 
respectively. A full course of AL consisted of 6 doses given twice daily ( $8 \mathrm{~h}$ apart on day 0 and 12 -h on days 1 and 2). Patients were observed for $30 \mathrm{~min}$ to ensure they did not vomit the study drugs. If vomiting occurred, a repeat dose was given after vomiting stopped. Any patient who persistently vomited the study medication was withdrawn and treated with quinine (injection/ intravenous) or artesunate injection according to the national guidelines for management of complicated and severe malaria [8]. Paracetamol was given to all patients with body temperature $\geq 38{ }^{\circ} \mathrm{C}$. All (morning and evening) doses were administered orally at the health facility under direct observation of a study nurse but without nutritional supplements. Patients living far from the study health facilities were retained in the wards for the evening and morning doses of the drugs, while those staying close were provided with transport to the facilities for the evening doses.

Follow-up was done for 28 days with scheduled visits on days $1,2,3,7,14,21$, and 28 or at any other time (unscheduled visit) when patients felt unwell. Parents/ guardians were informed and encouraged to bring their children back to the clinic whenever they were unwell without waiting for scheduled visits. Patients who did not show up for their scheduled visits by mid-day were visited at home by a member of the study team and asked to come to the health facility. If a patient had travelled and could not be traced for scheduled follow-up, he/she was classified as lost to follow-up. During the visits, both clinical and parasitological assessments were performed and DBS were also collected. Patients with recurrent infections occurring on day 7 and afterwards were treated with quinine (tablets, injection/intravenous) or artesunate injection based on clinical presentation as per WHO protocol [46].

\section{Safety assessment}

The safety of AL was monitored by both passive and active methods through interviews with parents/guardian and clinical/laboratory assessments during the 28 days of follow-up. This enabled investigators to capture and record adverse events (AEs) or severe adverse events (SAEs) that occurred after treatment. During scheduled visits, parents/guardians were directly interviewed and asked to report the occurrence, nature, and incidence of any events occurring at home between the follow-up visits. Clinicians took a history, observed patients, and performed a clinical examination during follow-up visits at the study sites. Laboratory tests were appropriately requested and done to determine and capture AEs/SAEs. The reported/captured events were recorded in respective case report forms for each follow-up visit. Any SAE occurring during the study was reported by the principal investigator to the sponsor (National Institute for Medical Research-NIMR), NMCP, and the Tanzanian Medical Research Coordinating Committee (MRCC) of NIMR (which is the Tanzanian national ethics committee) within $24 \mathrm{~h}$ of its occurrence. Reporting of SAEs was done regardless of whether the principal investigator considered the events to be related to the investigated drug or not. Patients with AEs or SAEs were thoroughly assessed and managed accordingly, and the events were also assessed to determine their association with the study drugs. According to the WHO protocol [46], an AE was defined as any unfavourable, unintended sign, symptom, syndrome or disease that develops or worsens with the use of a medicinal product, regardless of whether it is related to the medicinal product. An SAE was also defined as any untoward medical occurrence that at any dose may lead to either death, life threatening condition, hospitalization or prolongation of hospitalization, significant disability, or incapacity.

\section{Sample processing and molecular analysis}

Molecular analysis was performed on all samples collected upon enrolment (day 0) and during follow up in the case of treatment failure. Parasite genomic DNA was extracted from DBS using QIAamp blood mini-kits (Qiagen $\mathrm{GmbH}$, Hilden, Germany) according to the manufacturer's instructions. Molecular markers of anti-malarial drug resistance and microsatellite markers were analysed at the Centers for Disease Control and Prevention (CDC) Malaria Laboratory in Atlanta, USA. Sanger sequences generated in this study were analysed using the Geneious software package (Biomatters, Inc., San Francisco, CA). Raw sequence reads were cleaned using default settings, and reads with high-quality scores (the percentage of high-quality bases) below $70 \%$ were discarded from further analysis. The $P f k 13$ propeller domain (codon positions: 440-600) and Pfmdr1 (codon positions: 86, 184 and 1246) were analysed for SNPs. SNPs were called only if they fit the following criteria: (i) they were found on both the forward and reverse reads, (ii) they had a $p$ - value of $<0.0001$ ( $p$-value represents the probability of a sequencing error resulting in observing bases with at least the given sum of qualities), and (iii) they had a minimum strand bias $p$-value of $<0.0005$ when exceeding $65 \%$ strand bias, as some errors from sequencing machines are more likely to happen on nearby upstream bases. Mixed-infection and/or heterozygous calls were excluded from the analysis. The 3D7 Pfk13 and Pfmdr1 were used as reference sequences. Detection of $P f m d r 1$ copy number variants was performed using an Agilent Mx3005 real-time PCR machine (Agilent Technologies, California, USA) according to previously described protocols $[47,48]$. 
Samples from 65 patients with recurrent infections were analysed to determine genetic diversity in the study populations using six neutral microsatellite markers (TA1 on Chromosome 6, Poly- $\alpha$ on chromosome 4, PfPK2 on chromosome 12, 2490 on chromosome 10, C2M34-313 on chromosome 2 and C2M69-383 on chromosome 3) by nested PCR for all except C2M34-313 and C2M69383 (which were analysed with a single step PCR). Fragment size was measured by capillary electrophoresis on ABI 3033 (Applied Biosystems) and scored using GeneMarker $^{\circledR}$ V2.6.3 (SoftGenetic, LLC, PA, USA) [48]). Paired samples (day 0 and parasites collected on or after day 7) were analysed to distinguish recrudescent from new infections by comparing alleles of samples collected at enrolment (on day 0) with those collected on the day of recurrent infection as previously described $[49,50]$. A recrudescent infection was confirmed if there was a perfect match of alleles at all successfully genotyped microsatellite markers while any mismatch was reported as a new infection. Cases with genotyping failure in either day 0 or recurrent samples (or both) at all markers were reported as non-determined (unknown PCR) and excluded from analysis of PCR corrected treatment outcome.

\section{Outcome classification}

The primary end point was PCR corrected parasitological cure on day 28 as per WHO protocol [46], while secondary end points included parasitaemia on day 3 post-treatment, occurrence of AEs/SAEs, and molecular markers of drug resistance in $P f m d r 1$ and $P f k 13$ genes. Treatment outcomes were classified as either early treatment failure (ETF), late clinical failure (LCF), late parasitological failure (LPF), or adequate clinical and parasitological response (ACPR) before and after PCR correction; based on per protocol method and Kaplan-Meier analysis.

\section{Ethical considerations}

Ethical clearance was obtained from MRCC of NIMR, while permission to conduct the study at the health facilities was sought in writing from the relevant regional and district medical authorities. Ethical clearance form CDC was not required because the assessments done at the CDC Malaria Laboratory, using samples without linked identifiers, were determined by the CDC Center of Global Health's Human Research Protection Coordinator to not constitute engagement in human subjects' research. Detailed information of the study and benefits as well as its risks were explained to each study participant. Oral and written informed consent were obtained from parents or guardians of all patients before they were screened for possible inclusion into the study. The study was retrospectively registered at ClinicalTrials.gov, No. NCT03387631 (on 2nd January 2018) because of problems within the system which did not allow to register it before the study was launched.

\section{Data management and analysis}

Data were entered into a Microsoft Access database at the study sites followed by a second entry which was done centrally at NIMR Tanga Centre after the end of data collection. The data were later validated, cleaned, and analysed using STATA for Windows version 11 (STATA Corporation; TX, USA). Descriptive statistics such as percentages, mean, median, standard deviation, and range were reported as appropriate. In order to automatically generate the treatment outcomes (based on per protocol and Kaplan-Meier analysis), the data were appropriately formatted and transferred to the WHO Excel software template [51]. Baseline characteristics, primary outcomes, and secondary outcomes were compared among the four sites. Continuous variables, such as $\log _{10}$ transformed parasite density at enrolment and age, among the four sites were compared using $t$ test or analysis of variance-ANOVA (for normally distributed data) or Mann-Whitney U/Kruskal-Wallis test (nonparametric tests for non-normally distributed data). The prevalence of different haplotypes or SNPs in the Pfmrd1 and $P f k 13$ genes as well as $P f m d r 1$ copy numbers were reported. For the Pfmrd1 gene, the analysis focused on the three SNPs (N86ㅁ, Y184프 and D1246Y) and their corresponding haplotypes (particularly NFD), which have been associated with reduced susceptibility to lumefantrine $[28,29]$. Logistic regression was used to assess the odds of selection from lumefantrine sensitive ( $\underline{\mathbf{Y}} \underline{\mathbf{Y}})$ to resistant haplotype (NFD) on day zero and day of recurrence with adjustment for age of patients and the study site (to account for potential differences in parasite populations attributable to variations in geographic locations). For all statistical tests and comparisons, a p-value $<0.05$ (two tailed) was considered to be significant.

\section{Results}

\section{Baseline characteristics of enrolled children}

A total of 963 children were screened between April and October 2016. All sites recruited 88 children except Kibaha, which enrolled 80, making a total of 344 enrolled at the four sites (Fig. 2). Table 1 shows the baseline characteristics of enrolled patients. More boys (57.0\%) were enrolled than girls, but the difference among the sites was not significant $(\mathrm{p}=0.962)$. Overall, Mlimba recruited significantly younger children compared to other sites, while children with the highest age were recruited at Kibaha $(\mathrm{p}<0.001)$. The proportion of children under 5 years of age recruited at the two sites of Kibaha and Mkuzi was significantly lower compared to Mlimba and Ujiji $(\mathrm{p}<0.001)$. The average axillary 


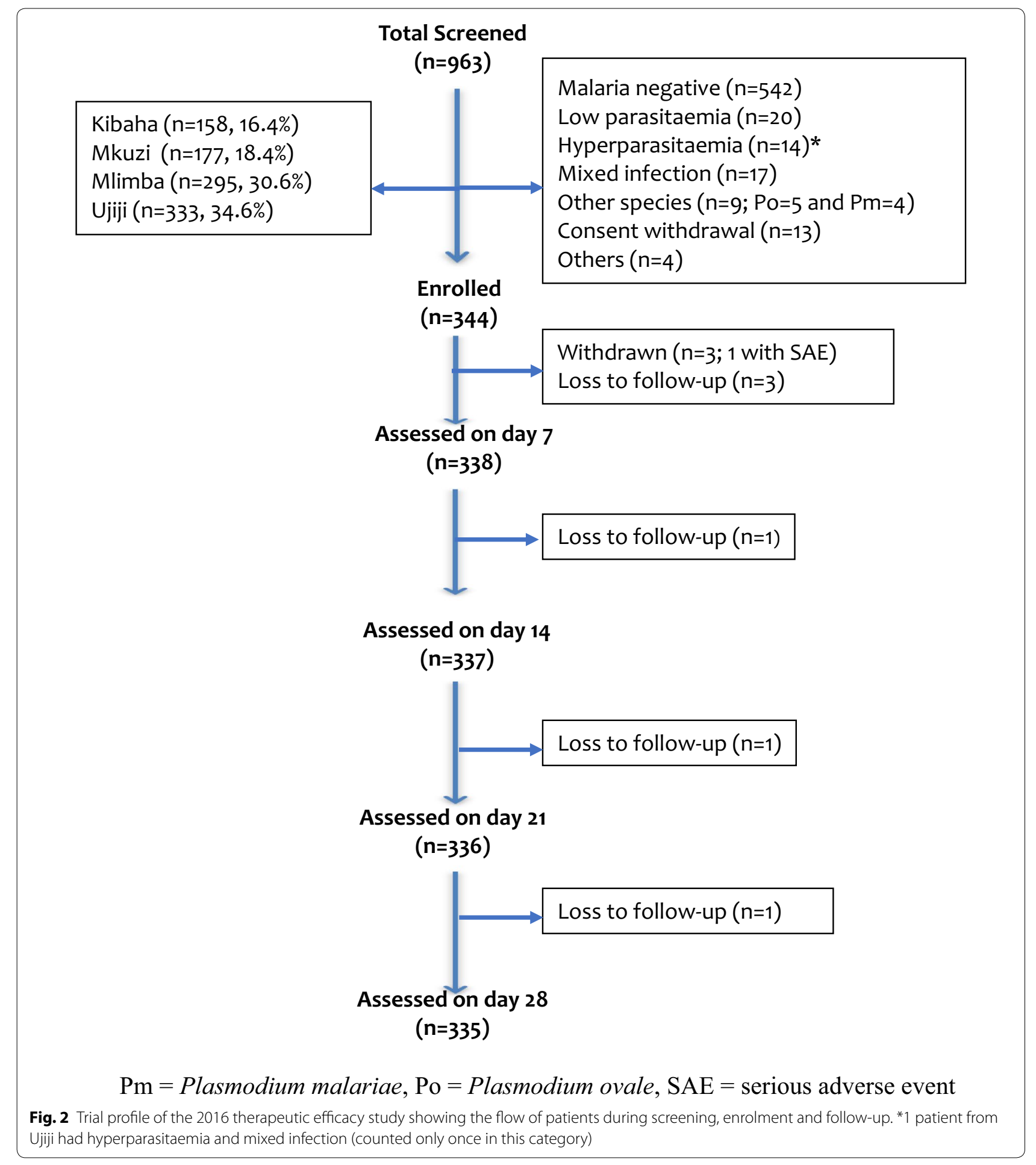

temperature recorded at enrolment (on day 0) was similar at all sites. The geometric mean parasite density (asexual parasites/ $\mu$ l) was significantly higher at Ujiji $(\mathrm{p}<0.001)$ compared to the other sites; patients enrolled at Kibaha had the lowest parasitaemia (Table 1).

\section{Efficacy outcomes}

Among the 344 patients enrolled, six (1.7\%) were lost to follow-up and three (0.9\%) withdrew, leaving 335 (97.4\%) patients that reached the study end points who were used in per protocol analysis (Fig. 1 and Table 2). In the 
Kaplan-Meier analysis, lost and withdrawn cases were included in the analysis until the last day seen. Based on the PCR uncorrected data, one patient (from Ujiji site) had ETF (0.3\%), 21 (6.3\%) had LCF, 47 (14.0\%) had LPF, and 266 (79.4\%) had ACPR (Table 2). After PCR correction, only one patient $(0.4 \%)$ had LCF, with a recrudescent infection reported on day 28; together with one patient with ETF (0.4\%), the overall PCR corrected ACPR was 99.3\% ( $\geq 98.4 \%$ at each site). Eleven (3.2\%) patients had parasitaemia on day 3 post-treatment, and no patients from Kibaha had parasites on day 3 while the highest positivity rates $(5.7 \%)$ was reported at Mkuzi (Table 2 ).

Table 1 Numbers of patients screened and baseline characteristics of patients enrolled in the therapeutic efficacy study at four sentinel sites in 2016

\begin{tabular}{|c|c|c|c|c|c|}
\hline Variable & Kibaha & Mkuzi & Mlimba & Ujiji & Overall \\
\hline Screened & 158 & 177 & 295 & 333 & 963 \\
\hline Enrolled (\%) & $80(50.6)$ & $88(49.7)$ & $88(30.3)$ & $88(26.4)$ & $344(35.7)$ \\
\hline Age in years, mean $(S D)^{a}$ & $6.5(2.7)$ & $5.7(2.8)$ & $3.4(2.3)$ & $4.6(2.9)$ & $5.0(2.9)$ \\
\hline $\begin{array}{l}\text { Children< <ive years of age, } \\
n(\%) \dagger\end{array}$ & $29(36.3)$ & $36(40.9)$ & $66(75.0)$ & $58(65.9)$ & $189(54.9)$ \\
\hline Sex (male), n (\%) & $46(57.5 \%)$ & $49(55.7 \%)$ & $49(55.7 \%)$ & $52(59.1 \%)$ & $196(57.0)$ \\
\hline Weight $(\mathrm{kg})$, mean $(\mathrm{SD})^{\mathrm{b}}$ & $19.1(5.6)$ & $17.4(5.0)$ & $13.1(4.3)$ & $14.9(5.1)$ & $16.1(5.5)$ \\
\hline Height in cm, median $(\mathrm{IQR})^{\mathrm{c}}$ & $114.0(98.5,128.3)$ & $111.0(92.1,121.0)$ & $85.5(77.0,99.8)$ & $97.0(86.0,111.8)$ & $100.0(85.0,117.8)$ \\
\hline Temperature in ${ }^{\circ} \mathrm{C}$, mean (SD) & $37.8(1.4)$ & $37.7(1.1)$ & $37.6(1.3)$ & $37.7(1.4)$ & $37.7(1.3)$ \\
\hline Parasitaemia* $\left(95 \%\right.$ Cl) ${ }^{a}$ & $9403(5901-14,984)$ & $32,357(23,393-44,756)$ & $27,720(20,706-37,108)$ & $41,106(30,356-55,664)$ & $24,806(20,701-29,726)$ \\
\hline
\end{tabular}

${ }^{\circ} \mathrm{C}$ : degree Celsius; parasitaemia*: geometric mean parasite density (asexual parasites/ $\mu$ l); IQR: interquartile range; $\mathrm{n}$ : number of patients; SD: standard deviation; $95 \% \mathrm{Cl}: 95 \%$ confidence interval. $+p<0.001$; ${ }^{a}$ the mean was significantly different between all paired comparison of the sites ( $p<<0.031$ ) except for Kibaha vs Mkuzi $(p=0.300)$; ${ }^{b}$ the mean weight was significantly different between all paired comparison of the sites $(p \leq<0.005)$ except for Kibaha vs Mkuzi $(p=0.200)$, and Mlimba vs Ujiji $(p=0.120){ }^{c}$ the mean height was significantly different between all paired comparison of the sites $(p<0.001)$ except for Kibaha vs Mkuzi $(p=0.138)$, and Mkuzi vs Ujiji $(p=0.068)$

Table 2 Measures of therapeutic efficacy of AL before and after PCR correction

\begin{tabular}{|c|c|c|c|c|c|}
\hline Outcome & $\begin{array}{l}\text { Kibaha } \\
\text { n (\%; } 95 \% \mathrm{CI})\end{array}$ & $\begin{array}{l}\text { Mlimba } \\
\text { n (\%; } 95 \% \mathrm{Cl})\end{array}$ & $\begin{array}{l}\text { Mkuzi } \\
\text { n (\%; 95\% Cl) }\end{array}$ & $\begin{array}{l}\text { Ujiji } \\
\text { n (\%; 95\% Cl) }\end{array}$ & $\begin{array}{l}\text { Total } \\
\text { n (\%; 95\% Cl) }\end{array}$ \\
\hline \multicolumn{6}{|l|}{ PCR uncorrected } \\
\hline Parasitaemia on day 3 & $0(0 ; 0-4.5)$ & $2(2.3 ; 0.3-8.1)$ & $5(5.7 ; 1.9-12.9)$ & $4(4.7 ; 1.3-11.5)$ & $11(3.3 ; 1.7-5.8)$ \\
\hline ETF & $0(0 ; 0-4.7)$ & $0(0 ; 0-4.2)$ & $0(0 ; 0-4.2)$ & $1(1.1 \% ; 0-6.2)$ & $1(0.3 ; 0-1.7)$ \\
\hline LCF & $12(15.8 ; 8.4-26.0)$ & $2(2.3 ; 0.3-8.1)$ & $3(3.5 ; 0.7-9.9)$ & $4(4.6 ; 1.3-11.4)$ & $21(6.3 ; 3.9-9.4)$ \\
\hline LPF & $6(7.9 ; 3.0-16.4)$ & $11(12.8 ; 6.6-21.7)$ & $11(12.8 ; 6.6-21.7)$ & $19(21.8 ; 13.7-32.0)$ & $47(14.0 ; 10.5-18.2)$ \\
\hline ACPR & $58(76.3 ; 65.2-85.3)$ & $73(84.9 ; 75.5-91.7)$ & $72(83.7 ; 74.2-90.8)$ & $63(72.4 ; 61.8-81.5)$ & $266(79.4 ; 74.7-83.9)$ \\
\hline Total for per protocol & 76 & 86 & 86 & 87 & 335 \\
\hline Withdrawn & $0(0.0 \%)$ & $1(1.1)$ & $1(1.1)$ & $1(1.1)$ & $3(0.9 \%)$ \\
\hline Lost to follow-up & $4(5.0 \%)$ & $1(1.1)$ & $1(1.1)$ & $0(0)$ & $6(1.7 \%)$ \\
\hline Total at baseline & 80 & 88 & 88 & 88 & 344 \\
\hline \multicolumn{6}{|l|}{ PCR corrected } \\
\hline ETF & $0(0 ; 0-6.2)$ & $0(0 ; 0-4.9)$ & $0(0 ; 0-4.9)$ & $1(1.6 ;)$ & $1(0.4 ; 0-2.1)$ \\
\hline LCF & $0(0 ; 0-6.2)$ & $0(0 ; 0-4.9)$ & $1(1.4:)$ & $0(0 ; 0-5.6)$ & $1(0.4 ; 0-2.1)$ \\
\hline LPF & $0(0 ; 0-6.2)$ & $0(0 ; 0-4.9)$ & $0(0 ; 0-4.9)$ & $0(0 ; 0-5.6)$ & $0(0 ; 0-1.4)$ \\
\hline ACPR & $58(100 ; 93.8-100)$ & $73(100 ; 95.1-100)$ & $72(98.6 ; 92.6-100)$ & $63(98.4 ; 91.6-100)$ & $266(99.3 ; 97.3-99.9)$ \\
\hline Total for per protocol & 58 & 73 & 73 & 64 & 268 \\
\hline Withdrawn/lost to follow-up & $4(5.0 \%)$ & $2(2.2)$ & $2(2.2)$ & $1(1.1)$ & $9(2.6)$ \\
\hline Re-infection & $15(18.8)$ & $11(12.5)$ & $9(10.2)$ & $20(22.7)$ & $55(16.0)$ \\
\hline Unknown PCR & $3(3.4)$ & $2(0.6)$ & $4(1.2)$ & $3(3.4)$ & $12(3.5)$ \\
\hline Total at baseline & 80 & 88 & 88 & 88 & 344 \\
\hline KM cumulative success rate & $58(100)$ & $73(100)$ & $72(98.6)$ & $63(98.4)$ & $266(99.3)$ \\
\hline
\end{tabular}

ACPR: adequate clinical and parasitological response; ETF: early treatment failure; LCF: late clinical failure; LPF: late parasitological failure; PP: number of patients involve in the per protocol analysis; KM: Kaplan-Meier

a The samples could not be resolved after PCR because of inconsistent PCR results 


\section{Safety outcomes}

The commonly reported adverse events included cough, abdominal pain, vomiting, and diarrhoea. Most events were reported at Mkuzi site (Table 3). The time periods when these events occurred during follow-up are presented in Supplemental Table 1. Two patients had SAEs on day 0 , including one patient (9 years, Female) from Mkuzi who died after the first dose of AL and a second patient (3.5 years, Male) from Ujiji who was hospitalized after the second dose of AL. Further assessment showed that the child from Ujiji was brought back with severe malaria, high fever (temperature $=39.5{ }^{\circ} \mathrm{C}$ ), vomiting, and convulsions after the second dose of AL. This child was treated with injectable artesunate (intravenous) and later transitioned to AL tablets and recovered completely. However, the child from Mkuzi succumbed to severe malaria with high fever (axillary temperature $=39.2{ }^{\circ} \mathrm{C}$ ) and convulsions after the first dose of AL and died immediately after arrival in the ward before any further treatment could be given. The child had no danger signs at enrolment and the initial parasitaemia was 16,160 asex$\mathrm{ual} / \mu \mathrm{l}$. The axillary temperature at screening was $37.1^{\circ} \mathrm{C}$ and the child walked by herself to the facility at the initial consultation. Post-mortem examination could not be performed because the deceased was buried the following day based on the family's decision. The actual cause of death was not established and could not be ascertained if it was associated with the medication.

\section{Molecular markers of anti-malarial drug resistance}

Out of 344 samples collected at enrolment (on day 0), 92.7\% $(\mathrm{n}=319)$ and $100 \%(\mathrm{n}=344)$ were successfully sequenced for $P f k 13$ and $P f m d r 1$, respectively. Seventeen samples (5.3\%) had mutations in the $P f k 13$ gene and only $6(1.9 \%)$ samples had non-synonymous mutations, of which none were similar to previously reported SNPs associated with artemisinin resistance. The six $P f k 13$ non-synonymous mutations included one patient from Mkuzi (R471ㅁ), two at Mlimba (A578 $\underline{\mathbf{S}}$ and E433므), and three at Ujiji (one with I416V and two with Q613E).
All patients with parasitaemia on day 3 post-treatment had infections with $P f k 13$ wild-type at enrolment. For Pfmdr1, codons N86Y Y Y184F and D1246ㅍ were analysed and the prevalence of the different SNPs are presented in Table 4. The N86 and D1246 polymorphisms were found at a prevalence of greater than $98.9 \%$ across the four sites. In comparison, the $184 \underline{\mathbf{F}}$ mutant was present between 34.1\% (Mlimba) and 46.6\% (Mkuzi) in all four sites (Table 4), the differences were not significant ( $\mathrm{p}>0.310)$. The NFD Pfmdr1 haplotype, which is possibly associated with decreased susceptibly to lumefantrine, was detected in $134(39.0 \%)$ samples, and its prevalence ranged from $33.0 \%$ in Mlimba to $45.5 \%$ at Mkuzi, but there were no significant differences $(\mathrm{p}=0.578)$ among the four sites (Fig. 3). Among 65 patients with recurrent infections (including 64 with new infections and one with a recrudescent infection), 39 (60.0\%) had NYD (wildtype) haplotype at enrolment and 15 (38.5\%) of these had selection to NFD haplotype (with mutations at codon 184ㅍ) during recurrent infections. Although patients with the NFD haplotype at baseline had more recurrent infections, the difference was not significant, even after adjusting for age and study site (adjusted OR $=1.17,95 \% \mathrm{CI}=0.66-2.05$, $\mathrm{p}=0.594)$. Both patients with PCR-corrected treat failure (one with ETF and the second with a recrudescent infection) had NFD haplotype at enrolment. The overall median day of recurrent infection was 21 days (interquartile range, 21-28) and this was similar among the

\begin{tabular}{|c|c|c|c|c|c|}
\hline SNP & Kibaha & Mkuzi & Mlimba & Ujiji & Total \\
\hline$N 86^{a}$ & 79 (98.8) & 87 (98.9) & 87 (98.9) & 88 (100.0) & 341 (99.1) \\
\hline$\underline{184 F}$ & $34(42.5)$ & 41 (46.6) & $30(34.1)$ & $32(36.4)$ & 137 (39.8) \\
\hline D1246 & $80(100.0)$ & 87 (98.9) & 87 (98.9) & 88 (100.0) & $342(99.4)$ \\
\hline
\end{tabular}

a One sample from Mlimba had an N86! mutation while the others with mutations at this SNP possessed N86ㅇ (2 samples, one each from Kibaha and Mkuzi)

Table 3 Number and proportion of patients with at least one adverse event

\begin{tabular}{|c|c|c|c|c|c|}
\hline Adverse events & Kibaha $(n=80)$ & Ujiji $(n=88)$ & Mkuzi (n=88) & Mlimba $(n=88)$ & Total $(n=344)$ \\
\hline Cough & $0(0 \%)$ & $12(13.6 \%)$ & $31(35.2 \%)$ & $1(1.1 \%)$ & $44(12.8 \%)$ \\
\hline Abdominal pain & $2(2.5 \%)$ & $1(1.1 \%)$ & $12(13.6 \%)$ & $0(0 \%)$ & $15(4.4 \%)$ \\
\hline Vomiting & $3(3.8 \%)$ & $0(0 \%)$ & $9(10.2 \%)$ & $1(1.1 \%)$ & 13 (3.8\%) \\
\hline Diarrhoea & $0(0 \%)$ & $0(0 \%)$ & $3(3.4 \%)$ & $1(1.1 \%)$ & $4(1.2 \%)$ \\
\hline Headache & $1(1.3 \%)$ & $0(0 \%)$ & $1(1.1 \%)$ & $0(0 \%)$ & $2(0.6 \%)$ \\
\hline Others & $0(0 \%)$ & $10(11.4 \%)$ & $5(3.4 \%)$ & $3(3.4 \%)$ & $18(5.2 \%)$ \\
\hline Total & $6(7.5 \%)$ & $23(26.1 \%)$ & 61 (69.3\%) & $6(6.8 \%)$ & 96 (27.9\%) \\
\hline
\end{tabular}



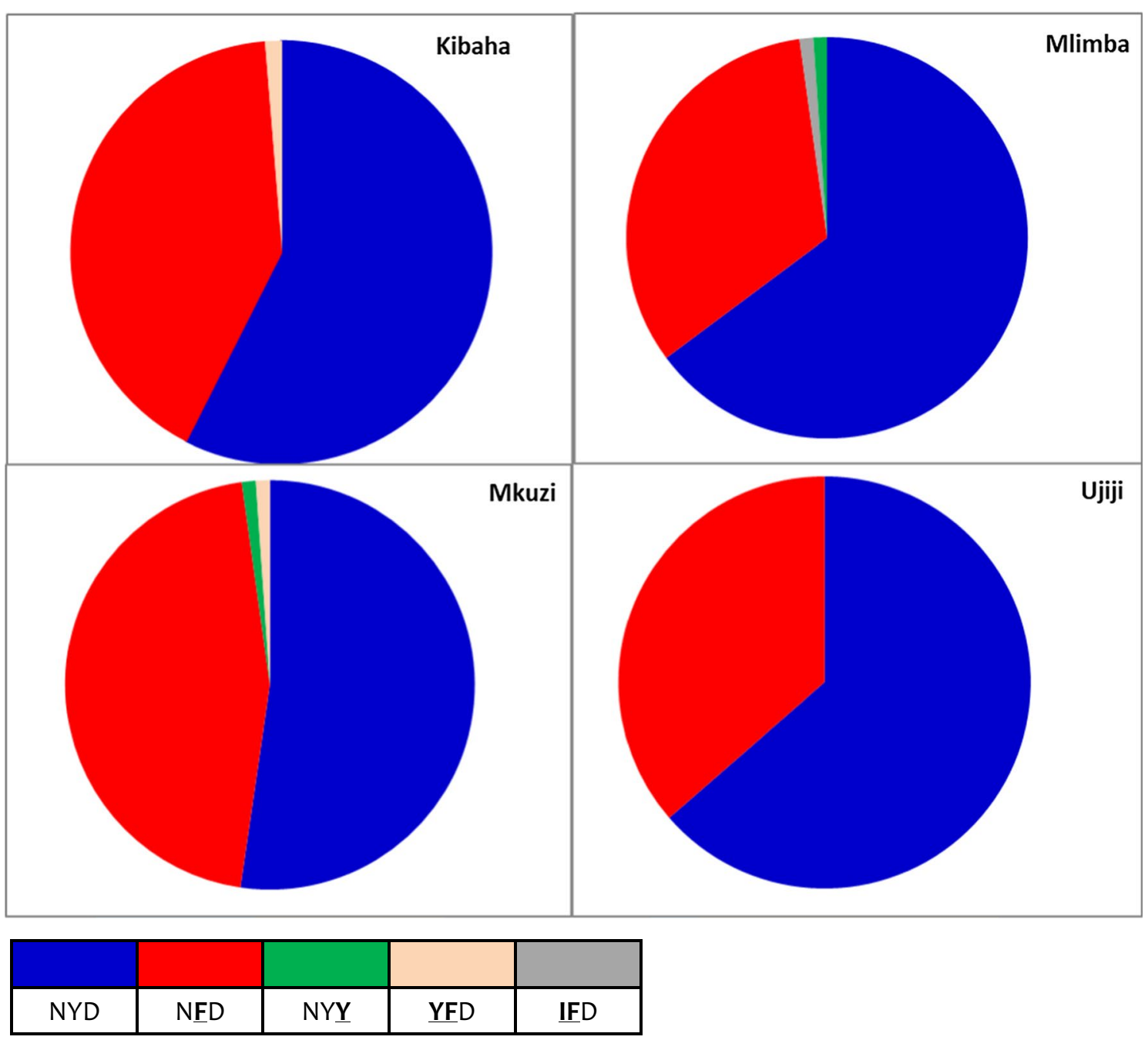

Fig. 3 Prevalence of different haplotypes in the Pfmdrl gene in baseline samples collected at enrolment of study patients

four sites. Recurrent infections among individuals with NFD haplotypes at enrolment had a median of 23 days compared to 21 days for NYD, but the difference was not statistically significant $(\mathrm{p}=0.417)$. Analysis of $P f m d r 1$ copy number variants was done on all 65 individuals with recurrent infections; all samples had a single copy of the gene.

\section{Discussion}

This study reports high efficacy of AL (PCR corrected ACPR $>98 \%$ ), suggesting that the ACT has retained its ability to treat uncomplicated malaria despite its use in Mainland Tanzania for more than 10 years. Previous studies conducted at these and other sites in Tanzania reported similarly high efficacies of AL [9-12]. However, given the high level of re-infections observed in the study areas, multiple interventions are warranted, including both curative and preventive approaches such as insecticide treated nets to reduce the burden of malaria. Studies conducted in different parts of Tanzania showed that about $30 \%$ of patients developed new infections during follow-up, and this was mainly attributed to high transmission of malaria [9]. Despite a decline in malaria transmission, which was reported from 2007 onwards [34-36, 39], this study still reported high rates of recurrent infections ranging from 15.1 to $27.6 \%$, with the highest rates at Ujiji. This could be attributed to a resurgence of malaria, which indeed was reported in 2016, when parasite prevalence increased from its 2012 level of $9.5 \%$ to $14.8 \%$ nationally, with similar increases in the study regions [34, 36].

This study also showed that AL had a safety profile comparable to previous studies and was well tolerated with minimal AEs. Most of the AEs were minor and mainly reported at the two sites of Mkuzi and Ujiji. Studies conducted in Tanzania $[9,12]$ and elsewhere in 
Africa $[18,19,52]$ reported similar safety profiles of AL when used for the treatment of uncomplicated falciparum malaria. A high number of cases reporting cough at Mkuzi could be attributed to weather conditions, which were relatively cold and rainy at the time of the study.

Two patients had serious adverse events on the day of presentation (day 0), with one child from Mkuzi dying after the first dose of AL. The cause of death was not established and its possible association with AL treatment could not be ascertained. A similar incident of death was reported in a TES which was conducted at Nagaga in 2012 [12], and such deaths reported in TES are unlikely to be caused by AL treatment. However, close monitoring of patients with a possibility of progressing to severe disease due to danger signs or high parasitaemia (> 100,000 asexual parasites/ $\mu$ l) has been recommended and will be undertaken in future studies.

Analysis of $P f k 13$, a molecular marker of artemisinin resistance [25], revealed that $17(5.3 \%)$ samples had mutations at different locations of the gene, although the majority were synonymous changes and none of the non-synonymous mutations were similar to those reported to be associated with artemisinin resistance in Southeast Asia. The observed prevalence of $P f k 13$ nonsynonymous mutations in this study was higher than in a previous study which reported a prevalence of $1.2 \%$ [53]. All patients with parasites on day 3 had infections with parasites possessing wild type $P f k 13$ at enrolment. These findings do not suggest that artemisinin resistance has emerged in Tanzania [21-23] and other malaria endemic areas in Africa, as previously reported [20]. However, they suggest that $P f k 13$ mutations might be increasingly accumulating in Tanzanian parasite populations and thus continued surveillance will be required to monitor the trends of $P f k 13$ polymorphisms and their possible association with reduced efficacy of artemisinins.

Analysis of the Pfmdr1 gene showed that $39.0 \%$ of the parasites tested had the N86/Y184羊/D1246 (NFD) haplotype, a combination associated with reduced susceptibility to lumefantrine in some studies [54]. This combination of Pfmdr1 SNPs had higher prevalence than what was reported by previous studies in Mainland Tanzania [55] and Zanzibar [56]; however, this was not associated with increased risk of treatment failure following AL treatment. The significance of these findings is unclear but the SNPs may reflect the long-term use of AL in Tanzania and should be monitored in future studies.

Although studies have associated elevated $P f m d r 1$ copy number with reduced susceptibility to lumefantrine [30, $47,57]$, recent pooled analysis, however, failed to show such association (28) but supported an association with reduced sensitivity to mefloquine. The parasites analysed in this study had only one copy of Pfmdr1 gene. This could be related to the fact that mefloquine has never been used as a routine first-line therapy in the study areas.

\section{Conclusion}

The findings of this study showed that the efficacy of AL remains high in Tanzania despite the use of this combination for more than 10 years. The safety of $\mathrm{AL}$ was consistent with previous reports and no known artemisinin-resistance $P f k 13$ mutations or amplification of the $P f m d r 1$ gene were identified. A high prevalence of a Pfmdr1 haplotype was detected but corresponding subjects with treatment failure were not found. Continued TESs and monitoring of markers of resistance to artemisinin and partner drugs is critical and should be sustained to facilitate early detection of resistant parasites and to inform evidence-based malaria treatment policies.

\begin{abstract}
Abbreviations
ACPR: adequate clinical and parasitological response; ACT: artemisinin-based combination therapy; AL: artemether-lumefantrine; ASAQ: artesunate + amodiaquine; AS + SP: artesunate + sulfadoxine/pyrimethamine; CDC: Centers for Disease Control and Prevention; DBS: dried blood spot; DNA: deoxyribonucleic acid; DP: Dihydroartemisinin-Piperaquine; ETF: early treatment failure; LCF: late clinical failure; LPF: late parasitological failure; MRCC: Medical Research Coordinating Committee; PARMA: US PMI-supported Antimalarial Resistance Monitoring in Africa Network; Pfk 13: Plasmodium falciparum Kelch 13 gene; Pfmdr1: Plasmodium falciparum multi-drug resistance 1 gene; PMI: US President's Malaria Initiative; NIMR: National Institute for Medical Research; NMCP: National Malaria Control Programme; PCR: polymerase chain reaction; SNP: single nucleotide polymorphism; TES: therapeutic efficacy study; USA: United States of America; WHO: World Health Organization.
\end{abstract}

\section{Authors' contributions}

DSI, CIM, BN, AMK, MKM, EK, RAK, FM, AM, RM, SM, FC, LP, GG, RN and MW designed the study and took part in field data collection. DSI, CIM and FF supervised data collection, overall implementation of the study and performed data analysis. DSI, CIM and MW managed, cleaned and analysed clinical data. DSI, ET, NWL, MV, ESH and VU took part and/or supervised molecular analysis of samples and data management. TM provided technical and logistic support and was involved in project management at PATH, while CAK was involved in planning and supervision of field work. DSI and CIM wrote the manuscript with the support of MW. All authors read and approved the final manuscript.

\footnotetext{
Author details

${ }^{1}$ National Institute for Medical Research, Tanga Research Centre, Tanga, Tanzania. ${ }^{2}$ Malaria Branch, Division of Parasitic Diseases and Malaria, Centers for Disease Control and Prevention, Atlanta, GA, USA. ${ }^{3}$ Department of Parasitology, School of Public Health, Muhimbili University of Health and Allied Sciences, Dar es Salaam, Tanzania. ${ }^{4}$ Department of Women's and Children's Health, International Maternal and Child Health (IMCH), Uppsala University, Uppsala, Sweden. ${ }^{5}$ Ifakara Health Institute, Dar es Salaam, Tanzania. ${ }^{6}$ Catholic University of Health and Allied Sciences/Bugando Medical Centre, Mwanza, Tanzania. ${ }^{7}$ Kilimanjaro Christian Medical Centre/Kilimanjaro Christian Medical University College, Moshi, Tanzania. ${ }^{8}$ National Malaria Control Programme, Ocean Road/Luthuli Avenue (NIMR Complex), Dar es Salaam, Tanzania. ${ }^{9}$ U.S. President's Malaria Initiative, U.S. Agency for International Development, U.S. Embassy, Dar es Salaam, Tanzania. ${ }^{10}$ World Health Organization Country Office, Dar es Salaam, Tanzania. ${ }^{11}$ HIV Vaccine Trials Network, Fred Hutch Cancer Research Center, Seattle, WA, USA. ${ }^{12}$ U.S. President's Malaria Initiative, U.S.
} 
Agency for International Development, Washington, DC, USA. ${ }^{13}$ Global Malaria Programme, World Health Organization, 20 Avenue Appia, 1211 Geneva 27, Switzerland. ${ }^{14}$ U.S. President's Malaria Initiative, Centers for Disease Control and Prevention, Atlanta, GA, USA. ${ }^{15}$ Present Address: Gothenburg University, Gothenburg, Sweden.

\section{Acknowledgements}

Authors wish to thank parents/guardians of children for taking part in the study. They are grateful to health facilities' staff, stakeholders, and colleagues from implementing partners and local health authorities for their support. Molecular analysis was undertaken at CDC with the support of The US President's Malaria Initiative-supported Antimalarial Resistance Monitoring in Africa (PARMA) Network. Technical and logistic support provided by the CDC, USAID/PARMA, and NIMR teams is highly appreciated. Test drugs and filter papers were provided by WHO through its Global Malaria Programme. Thanks to PATH teams (Seattle, WA and Washington DC, USA) for financial and logistics support. This study was sponsored by NIMR as the host institution of TES coordination on behalf of the TES Taskforce. Permission to publish this paper has been granted by the Director General of NIMR.

\section{Competing interests}

The authors declare that they have no competing interests.

\section{Availability of data}

The data set from this study has been shared with WHO as its part of global monitoring of TES. The data are available and can be shared upon reasonable request to NMCP through the corresponding author.

\section{Consent for publication}

Not applicable.

\section{Disclaimer}

Ritha Njau is a staff member of the World Health Organization and Marian Warsame is a recently retired staff of the World Health Organization. They alone are responsible for the views expressed in this publication, which do not necessarily represent the decisions, policy or views of the World Health Organization. Furthermore, the findings and conclusions in this paper are those of the authors and do not necessarily represent the official position of the US Centers for Disease Control and Prevention.

\section{Ethical approval and consent to participate}

This study was approved by the MRCC of NIMR and permission to conduct the study at the four sites was obtained from the district medical authorities of respective districts. Parents/guardians of all study participants signed an informed consent before enrolment.

\section{Funding}

This study was funded by USAID under the US President's Malaria Initiative through MalariaCare (PATH).

\section{Publisher's Note}

Springer Nature remains neutral with regard to jurisdictional claims in published maps and institutional affiliations.

Received: 14 January 2019 Accepted: 14 March 2019

Published online: 21 March 2019

\section{References}

1. WHO. World malaria report 2018. Geneva: World Health Organization; 2018.

2. Snow RW, Sartorius B, Kyalo D, Maina J, Amratia P, Mundia CW, et al. The prevalence of Plasmodium falciparum in sub-Saharan Africa since 1900. Nature. 2017;550:515-8.

3. WHO. World malaria report 2015. Geneva: World Health Organization; 2015.

4. WHO. World malaria report 2016. Geneva: World Health Organisation; 2016.
5. WHO. Guidelines for the treatment of malaria. 2nd ed. Geneva: World Health Organisation; 2010.

6. WHO. Guidelines for the treatment of malaria. 3rd ed. Geneva: World Health Organisation; 2015.

7. Ministry of Health and Social Welfare. National guidelines for malaria diagnosis and treatment. In: A. Mwita, F. Molten, (Eds.) Dar es Salam: United Republic of Tanzania, 2006. https://www.who.int/malaria/publi cations/atoz/9789241549127/en/

8. Ministry of Health and Social Welfare. National guidelines for diagnosis and treatment of malaria. Dar es Salam: United Republic of Tanzania; 2014.

9. Shayo A, Buza J, Ishengoma DS. Monitoring of efficacy and safety of artemisinin-based anti-malarials for treatment of uncomplicated malaria: a review of evidence of implementation of anti-malarial therapeutic efficacy trials in Tanzania. Malar J. 2015;14:135.

10. Mwaiswelo R, Ngasala B, Gil JP, Malmberg M, Jovel I, Xu W, et al. Sustained high cure rate of artemether-lumefantrine against uncomplicated Plasmodium falciparum malaria after 8 years of its wide-scale use in Bagamoyo district, Tanzania. Am J Trop Med Hyg. 2017;97:526-32.

11. Mandara Cl, Kavishe RA, Gesase S, Mghamba J, Ngadaya E, Mmbuji P, et al. High efficacy of artemether-lumefantrine and dihydroartemisininpiperaquine for the treatment of uncomplicated falciparum malaria in Muheza and Kigoma Districts, Tanzania. Malar J. 2018;17:261.

12. Kakolwa MA, Mahende MK, Ishengoma DS, Mandara Cl, Ngasala B, Kamugisha E, et al. Efficacy and safety of artemisinin-based combination therapy, and molecular markers for artemisinin and piperaquine resistance in Mainland Tanzania. Malar J. 2018;17:369.

13. Agarwal A, McMorrow M, Onyango P, Otieno K, Odero C, Williamson $J$, et al. A randomized trial of artemether-lumefantrine and dihydroartemisinin-piperaquine in the treatment of uncomplicated malaria among children in western Kenya. Malar J. 2013;12:254.

14. Yeka A, Kigozi R, Conrad MD, Lugemwa M, Okui P, Katureebe C, et al. Artesunate/amodiaquine versus artemether/lumefantrine for the treatment of uncomplicated malaria in Uganda: a randomized trial. J Infect Dis. 2016:213:1134-42.

15. Zwang J, Olliaro P, Barennes H, Bonnet M, Brasseur P, Bukirwa H, et al. Efficacy of artesunate-amodiaquine for treating uncomplicated falciparum malaria in sub-Saharan Africa: a multi-centre analysis. Malar J. 2009;8:203.

16. Ndayiragije A, Niyungeko D, Karenzo J, Niyungeko E, Barutwanayo M, Ciza A, et al. Efficacy of therapeutic combinations with artemisinin derivatives in the treatment of non complicated malaria in Burundi. Trop Med Int Health. 2004;9:673-9 (in French).

17. Attaran A, Barnes Kl, Curtis C, D'Alessandro U, Fanello Cl, Galinski MR, et al. $\mathrm{WHO}$, the Global Fund, and medical malpractice in malaria treatment. Lancet. 2004;363:237-40.

18. Makanga M, Krudsood S. The clinical efficacy of artemether/lumefantrine (Coartem). Malar J. 2009;8(Suppl 1):S5.

19. Makanga M, Bassat Q, Falade CO, Premji ZG, Krudsood S, Hunt P, et al. Efficacy and safety of artemether-lumefantrine in the treatment of acute, uncomplicated Plasmodium falciparum malaria: a pooled analysis. Am J Trop Med Hyg. 2011;85:793-804.

20. WHO. Artemisinin and artemisinin-based combination resistance. Geneva: World Health Organization; 2017.

21. Ashley EA, Dhorda M, Fairhurst RM, Amaratunga C, Lim P, Suon S, et al. Spread of artemisinin resistance in Plasmodium falciparum malaria. N Engl J Med. 2014;371:411.

22. Leang R, Taylor WR, Bouth DM, Song L, Tarning J, Char MC, et al. Evidence of Plasmodium falciparum malaria multidrug resistance to artemisinin and piperaquine in Western Cambodia: dihydroartemisinin-piperaquine open-label multicenter clinical assessment. Antimicrob Agents Chemother. 2015;59:4719-26.

23. Tun KM, Imwong M, Lwin KM, Win AA, Hlaing TM, Hlaing T, et al. Spread of artemisinin-resistant Plasmodium falciparum in Myanmar: a cross-sectional survey of the K13 molecular marker. Lancet Infect Dis. 2015;15:415-21.

24. Amaratunga C, Lim P, Suon S, Sreng S, Mao S, Sopha C, et al. Dihydroartemisinin-piperaquine resistance in Plasmodium falciparum malaria in Cambodia: a multisite prospective cohort study. Lancet Infect Dis. 2016;16:357-65. 
25. Ariey F, Witkowski B, Amaratunga C, Beghain J, Langlois AC, Khim N, et al. A molecular marker of artemisinin-resistant Plasmodium falciparum malaria. Nature. 2014;505:50-5.

26. Straimer J, Gnadig NF, Witkowski B, Amaratunga C, Duru V, Ramadani AP, et al. Drug resistance. K13-propeller mutations confer artemisinin resistance in Plasmodium falciparum clinical isolates. Science. 2015;347:428-31.

27. WHO. Artemisinin resistance and artemisinin-based combination efficacy: status report. Geneva: World Health Organization; 2018.

28. Venkatesan M, Gadalla NB, Stepniewska K, Dahal P, Nsanzabana C, Moriera C, et al. Polymorphisms in Plasmodium falciparum chloroquine resistance transporter and multidrug resistance 1 genes: parasite risk factors that affect treatment outcomes for $P$. falciparum malaria after artemether-lumefantrine and artesunate-amodiaquine. Am J Trop Med Hyg. 2014;91:833-43.

29. Malmberg M, Ferreira PE, Tarning J, Ursing J, Ngasala B, Bjorkman A, et al. Plasmodium falciparum drug resistance phenotype as assessed by patient antimalarial drug levels and its association with pfmdr 1 polymorphisms. J Infect Dis. 2013;207:842-7.

30. Price RN, Uhlemann AC, van Vugt M, Brockman A, Hutagalung R, Nair S, et al. Molecular and pharmacological determinants of the therapeutic response to artemether-lumefantrine in multidrug-resistant Plasmodium falciparum malaria. Clin Infect Dis. 2006;42:1570-7.

31. East African Network for Monitoring Antimalarial Treatment (EANMAT). Monitoring antimalarial drug resistance within National Malaria Control Programmes: the EANMAT experience. Trop Med Int Health. 2001;6:891-8.

32. The efficacy of antimalarial monotherapies. sulphadoxine-pyrimethamine and amodiaquine in East Africa: implications for sub-regional policy. Trop Med Int Health. 2003;8:860-7.

33. Ministry of Health. National guidelines for malaria diagnosis and treatment 2000. Dar es Salaam: United Republic of Tanzania; 2000.

34. United Republic of Tanzana. Tanzania Demographic and Health Survey and Malaria Indicator Survey. Dar es Salaam: United Republic of Tanzania; 2016.

35. United Republic of Tanzania. Tanzania HIV/AIDS and Malaria indicator survey 2007/2008. Dar es Salaam: United Republic of Tanzania; 2008.

36. United Republic of Tanzania. Tanzania HIV/AIDS and Malaria Indicator Survey 2011-2012. Dar es Salaam: United Republic of Tanzania; 2014.

37. Ellman R, Maxwell C, Finch R, Shayo D. Malaria and anaemia at different altitudes in the Muheza district of Tanzania: childhood morbidity in relation to level of exposure to infection. Ann Trop Med Parasitol. 1998;92:741-53.

38. Segeja MD, Mmbando BP, Kamugisha ML, Akida JA, Savaeli ZX, Minja DT, et al. Prevalence of glucose-6-phosphate dehydrogenase deficiency and haemoglobin $\mathrm{S}$ in high and moderate malaria transmission areas of Muheza, north-eastern Tanzania. Tanzan J Health Res. 2008;10:9-13.

39. United Republic of Tanzania. Tanzania HIV and malaria indicator survey 2017. Dar es Salaam: United Republic of Tanzania; 2017.

40. Ishengoma DS, Shayo A, Mandara CI, Baraka V, Madebe RA, Ngatunga D, et al. The role of malaria rapid diagnostic tests in screening of patients to be enrolled in clinical trials in low malaria transmission settings. Health Syst Policy Res. 2016;3:2

41. Geubbels E, Amri S, Levira F, Schellenberg J, Masanja H, Nathan R. Health \& demographic surveillance system profile: the Ifakara rural and urban health and demographic surveillance system (Ifakara HDSS). Int J Epidemiol. 2015;44:848-61.

42. Kabanywanyi AM, Mwita A, Sumari D, Mandike R, Mugittu K, Abdulla S. Efficacy and safety of artemisinin-based antimalarial in the treatment of uncomplicated malaria in children in southern Tanzania. Malar J. 2007;6:146.
43. Mbugi EV, Mutayoba BM, Malisa AL, Balthazary ST, Nyambo TB, Mshinda $H$. Drug resistance to sulphadoxine-pyrimethamine in Plasmodium falciparum malaria in Mlimba, Tanzania. Malar J. 2006:5:94.

44. Smith T, Charlwood JD, Kihonda J, Mwankusye S, Billingsley P, Meuwissen $\mathrm{J}$, et al. Absence of seasonal variation in malaria parasitaemia in an area of intense seasonal transmission. Acta Trop. 1993;54:55-72.

45. WHO. Malaria surveillance, monitoring and evaluation: a reference manual. Geneva: World Health Organization; 2018.

46. WHO. Methods for surveillance of antimalarial drug efficacy. Geneva: World Health Organisation; 2009.

47. Price RN, Uhlemann AC, Brockman A, McGready R, Ashley E, Phaipun $L$, et al. Mefloquine resistance in Plasmodium falciparum and increased pfmdr1 gene copy number. Lancet. 2004;364:438-47.

48. Davlantes E, Dimbu PR, Ferreira CM, Florinda JM, Pode D, Felix J, et al. Efficacy and safety of artemether-lumefantrine, artesunate-amodiaquine, and dihydroartemisinin-piperaquine for the treatment of uncomplicated Plasmodium falciparum malaria in three provinces in Angola, 2017. Malar J. 2018;17:144.

49. Greenhouse B, Myrick A, Dokomajilar C, Woo JM, Carlson EJ, Rosenthal PJ, et al. Validation of microsatellite markers for use in genotyping polyclonal Plasmodium falciparum infections. Am J Trop Med Hyg. 2006;75:836-42.

50. Nyachieo A, Van OC, Laurent T, Dujardin JC, D'Alessandro U. Plasmodium falciparum genotyping by microsatellites as a method to distinguish between recrudescent and new infections. Am J Trop Med Hyg. 2005:73:210-3

51. World Health Organization. Tools for monitoring antimalarial drug efficacy: WHO data entry and analysis tool. 2017. Geneva: World Health Organization; 2018.

52. Makanga M, Premji Z, Falade C, Karbwang J, Mueller EA, Andriano K, et al. Efficacy and safety of the six-dose regimen of artemether-lumefantrine in pediatrics with uncomplicated Plasmodium falciparum malaria: a pooled analysis of individual patient data. Am J Trop Med Hyg. 2006;74:991-8.

53. Kamau E, Campino S, Amenga-Etego L, Drury E, Ishengoma D, Johnson K, et al. K13-propeller polymorphisms in Plasmodium falciparum parasites from sub-Saharan Africa. J Infect Dis. 2015;211:1352-5.

54. Happi CT, Gbotosho GO, Folarin OA, Sowunmi A, Hudson T, O’Neil M, et al. Selection of Plasmodium falciparum multidrug resistance gene 1 alleles in asexual stages and gametocytes by artemether-lumefantrine in Nigerian children with uncomplicated falciparum malaria. Antimicrob Agents Chemother. 2009;53:888-95.

55. Thomsen TT, Ishengoma DS, Mmbando BP, Lusingu JP, Vestergaard LS, Theander TG, et al. Prevalence of single nucleotide polymorphisms in the Plasmodium falciparum multidrug resistance gene (Pfmdr-1) in Korogwe District in Tanzania before and after introduction of artemisinin-based combination therapy. Am J Trop Med Hyg. 2011;85:979-83.

56. Morris U, Xu W, Msellem MI, Schwartz A, Abass A, Shakely D, et al. Characterising temporal trends in asymptomatic Plasmodium infections and transporter polymorphisms during transition from high to low transmission in Zanzibar, 2005-2013. Infect Genet Evol. 2015;33:110-7.

57. Lim P, Alker AP, Khim N, Shah NK, Incardona S, Doung S, et al. Pfmdr1 copy number and arteminisin derivatives combination therapy failure in falciparum malaria in Cambodia. Malar J. 2009;8:11.

Ready to submit your research? Choose BMC and benefit from:

- fast, convenient online submission

- thorough peer review by experienced researchers in your field

- rapid publication on acceptance

- support for research data, including large and complex data types

- gold Open Access which fosters wider collaboration and increased citations

- maximum visibility for your research: over $100 \mathrm{M}$ website views per year

At $\mathrm{BMC}$, research is always in progress.

Learn more biomedcentral.com/submissions 